The final publication is available at Springer via https:// doi.org/10.1007/978-3-030-17771-3_23

\title{
Towards a Practical and Cost-effective Water Monitoring System
}

\author{
João Marques ${ }^{1}$, Brígida Lopes ${ }^{1}$, Carlos Ferreira ${ }^{1,2}$, Henrique Pinho ${ }^{1.2}$, Manuel \\ Barros $^{1,2}$, Pedro Granchinho ${ }^{1.2}$, Pedro Neves ${ }^{1}$, \\ ${ }^{1}$ E.S.T.T., Polytechnic Institute of Tomar, Department of Engineering, \\ Quinta do Contador - Estrada da Serra, 2300-313 Tomar, Portugal \\ \{jomarques, bablopes, cferreira, hpinho, fmbarros, granchinho, pedroneves\}@ipt.pt \\ ${ }^{2}$ Smart Cities Research Center (Ci2), Polytechnic Institute of Tomar, \\ Quinta do Contador - Estrada da Serra, 2300-313 Tomar, Portugal \\ \{cferreira, hpinho, fmbarros, granchinho\}@ipt.pt
}

\begin{abstract}
In recent years, there has been increasing awareness of the preservation, protection and sustainable use of natural resources. Water resources, being one of the most important, face major threats due to contamination by pollutants of various types and origins. Maintaining the quality of water resources requires more robust, reliable and more frequent monitoring than traditional data collection techniques based on manual sampling methods. This article, which is the result of ongoing research, proposes a practical and cost-effective solution for a surface water monitoring system, using a robotics platform and cloud services. The proposed solution allows for scalability and will accommodate a wide range of end-user specifications. To allow for continuous operation in longer activities, the design of a versatile real-time water quality monitoring system should also take into consideration the question of its energy requirements and self-sufficiency.
\end{abstract}

Keywords: Water quality monitoring, wireless sensor networks, autonomous vehicles, environment protection, resources management, IoT, environmental robotics, cloud computing.

\section{Introduction}

Society needs fast and reliable environmental monitoring platforms. Conventional processes involve the manual collection at various points from a sampling network, followed by laboratory testing and analysis. These methods can be seen as ineffective, as they are cumbersome, time-consuming, and lack real-time results to promote a proactive response to water contamination sources [1].

This work provides an outline for developing a system that solves the need for monitoring the surface water quality in natural and artificial freshwater systems of different types, whilst mitigating the normal entry-level barriers for the academia, local authorities, businesses and general population. In future works, a requirements 
specification plan will be introduced, which will be necessary for developing a working prototype.

Two key areas of environmental monitoring are particularly important in the context of this work, especially those concerning surface water:

Sensor Networks. Environmental sensor networks (ESN) have recently emerged as a promising technology for monitoring aquatic environments [2]. ESNs consist of a small group of small networked devices that allow real-time monitoring of relevant variables at various fixed and predetermined points [3]. ESNs have the advantage of allowing data collection in multiple locations simultaneously [4] and with greater fidelity. A major limitation of ESNs is that they usually need to have fixed sampling locations [3] and therefore do not have the ability to self-reconfigure in response to unexpected events or to increase coverage in the area of interest.

Environmental Robotics. Standalone robots for environmental monitoring are a promising solution to overcome the limitations of ESNs, adding mobility and thus increasing their potential. Robot groups can collect data from several places simultaneously, allowing to dynamically increase the spatial-temporal area of data collection that would be impossible to achieve with a single robot or a network of static sensors [3]. Some examples of this technology include the ROAZ II system [5] developed at the Polytechnic Institute of Porto (ISEP) an and the THA1592 project implemented in the Polytechnic Institute of Tomar [6]. Unmanned Aerial Vehicles (USVs) are often unable to achieve continuous operation because of their limited movement and energy autonomy.

\section{Relationship to industrial and service systems}

To meet the growing global need for fresh water sources, cloud computing can help manage data and information related to water supply and quality. Unfortunately, industry is not leveraging this new technology as fast and as widely as possible. The democratization of solutions, are not always in the interest of turnkey solutions providers (e.g. Consultants).

The research team also believes that in order to achieve a truly resilient system, a more agile mindset is required to face future and unexpected challenges. Water quality management is also more complex than, and potentially as important to the future of mankind as air quality management.

In the coming years, as sources of fresh water become scarcer worldwide, and more entities compete ever more vigorously for this resource, different businesses will be challenged in different ways. What is certain is that all businesses will be affected either directly or indirectly by the coming changes. Scarcity usually encourages better management of resources or forces extinction due to inadequate or inappropriate responses. Cloud Computing-based environmental solutions represent the best way for companies to take ownership of their data, satisfy looming reporting requirements, and tackle their water quality management challenges. 


\section{Rapid prototyping and deployment for environmental robotics}

In this section, the components for a robotic water monitoring system archetype are described. In order to achieve fast development speeds, a simple but robust robotics platform was selected. Sensor integration and communication also needs to be addressed. For these reasons, cloud services were chosen to achieve fast deployment speeds and reliability.

Figure 1 presents, schematically, the three main functional blocks of the proposed system: the robotics and IoT hardware and the cloud-based data storage and retrieval. For simplicity, the two systems will be functioning separately in a complementary fashion. The robotics module will allow the vehicle to execute user-defined missions, while the IoT sub-system gathers and processes the data from the attached sensors.

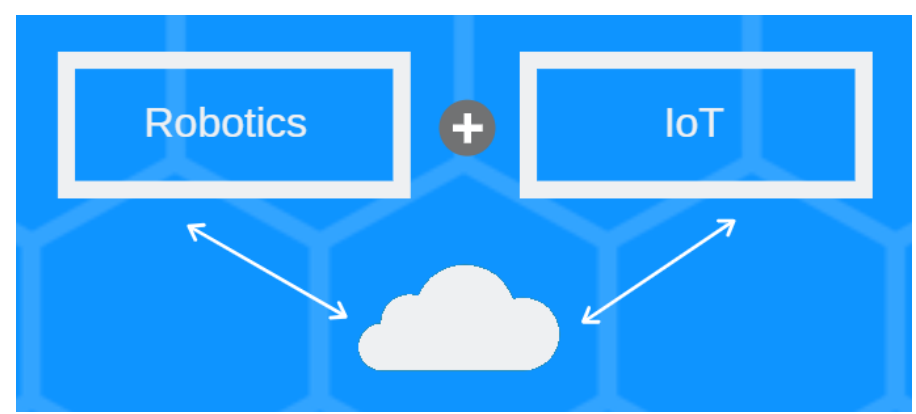

Fig. 1. Concept - Integration of robotics, IoT and cloud services technologies.

\subsection{Robotic platform overview}

The system consists of an autonomous, mobile and robotic monitoring system unit (called LIBÉLULA) with onboard sensors for the acquisition of water quality parameters $(\mathrm{pH}$, temperature, conductivity, etc.), a long-range wireless radio communication module and a microcontroller board with a GPS and compass unit. The control of the robotic platform will be based on a modular software architecture provided by an advanced, full-featured and reliable open source autopilot software, called Ardupilot. Ardupilot is open-source and has been developed over more than five years by a team of diverse professional engineers and computer scientists, being affirmed by the community as a well-tested and proven autopilot software. It provides services for navigation, localization, simulation, and integration with the well know robotic framework, ROS; which, in turn, allows remote monitoring of relevant water quality data and their dissemination in real time to a central server and cloud platforms [7]. 


\subsection{IoT Platform overview}

Cloud computing, on-demand computing or Internet based computing is a low-cost technology that offers fast and reliable processing, data storage, and several developer and analytical tools that allow both easy integration of physical devices and advanced data analysis. In this work, cloud computing services are to be used as the infrastructure backbone for the system as they provide fast deployment, continuous service, security and scalability. Although any provider of cloud services can be used, figure 2 shows a diagram consisting of an interconnected suite of applications made available from IBM, specifically for IoT.

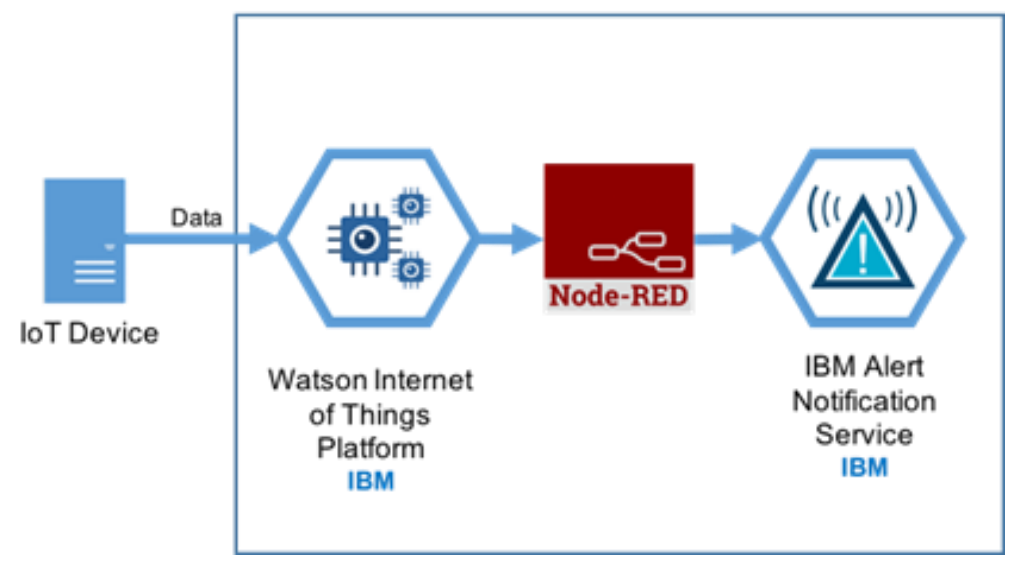

Fig. 2. Data flow for IoT system in the cloud (source: IBM)

\section{Water quality analysis and forecasting}

In order to adequately characterize the aquatic environment, the monitoring system needs to acquire a specific set of environmental data. Furthermore, to identify potential problems in water quality, some computational methods must be introduced to detect subtle deviations in the data. The most used of the general physical-chemical parameters are: water temperature, $\mathrm{pH}$, electrical conductivity, dissolved oxygen and total suspended solids. Following the theme of this work, whose main aim is to help the authorities in the "decision-making" process, different methods have been identified in the literature. Some of them are more in the field of traditional statistical techniques, such as principal component analysis (PCA), regression analysis, and cluster analysis [8] but in the age of never-ending streams of data there's also a growing tendency to use artificial intelligence algorithms and machine learning. A recent study has been made employing fuzzy logic, machine learning and adaptive filtering to successfully predict measurements a day ahead, as well as techniques to incorporate the window of past values in order to be able to make a more precise prediction [9]. 


\section{Energy management requirements}

Energy in mobile robots scenarios is an important issue. Fast charging methods and power converters should be used to power supply the system. Due to exposition to environmental conditions inductive contactless power transfer is a possible solution. It is a low maintenance solution and is safer by providing arc-free energy transfer.

Since the operation will normally be in remote locations, in terms of power, renewable energy sources should be considered in order to achieve autonomy for the system. Typically, probable power sources are water stream, converted to electricity through a hydrogenerator or, more generally, photovoltaic panels that generate power from sunlight. The harvested energy should be stored in a battery for permanent availability. Locating the charger station and precise docking are problems to be addressed.

\section{Building a case-study}

To validate the proposed platforms described in chapter 4 , field tests will be realized in the Castelo do Bode reservoir, one of Portugal's main water reserves. Figure 3 shows a sample mission, created using software for ground station control [10], which shall be executed by a robotic prototype developed by the research team from IPT. This is a very simple test and is intended as a proof of concept.

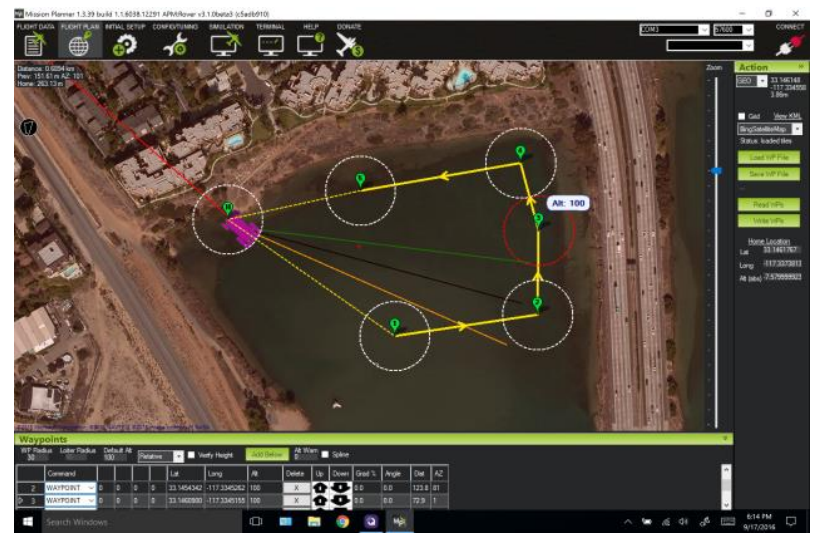

Fig. 3. Mission Planner software (source: Ardupilot)

In figure 4, a first prototype is shown for a low-cost, light-weight and durable catamaran type. In the future, work will be directed towards establishing a higher benchmark by developing a more robust working prototype, with the use of better materials as well as CAD and professional machining processes. During field testing, water parameters data is to be collected, stored and visualized using cloud services. Adding obstacle avoidance or the use of semantic segmentation for detection of 
navigable space, are desirable features that provide an important layer of intelligence and added mission autonomy. The proposed system has many potential applications and use-cases like surface water monitoring, wastewater management, research and development departments, small companies and individuals, local authorities and administrations, academic research, among others.

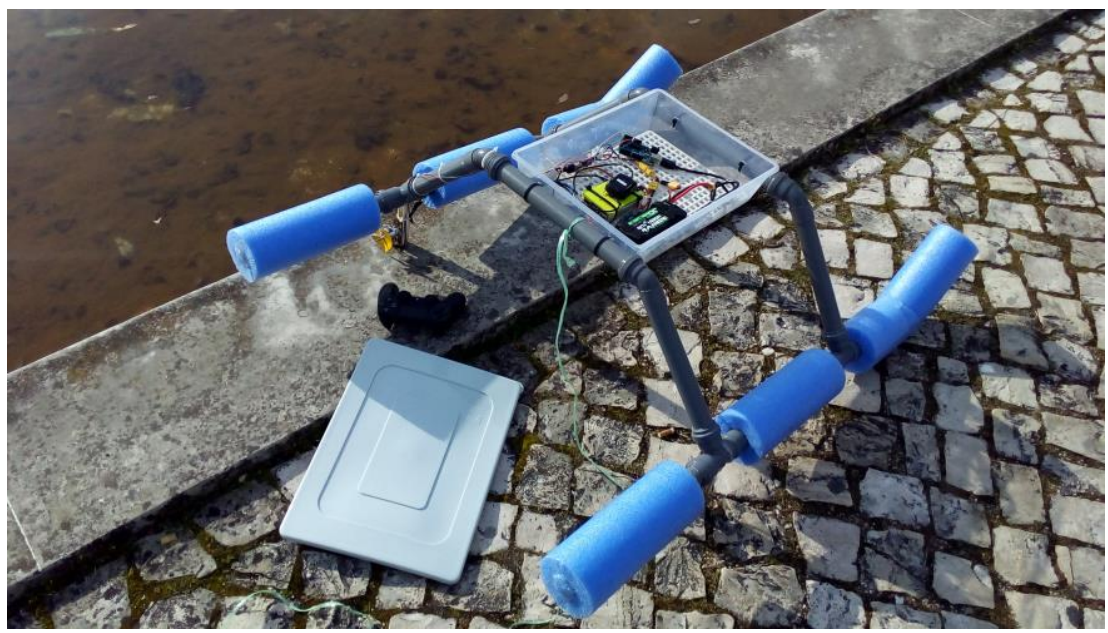

Fig. 4. Working prototype for a low-cost unmanned surface vehicle, developed by the research team from IPT.

\section{Conclusion}

The present work defines the technical requirements of a capable system of surface water quality monitoring for natural and artificial freshwater systems. A novel alternative is proposed, that builds on commercial and community (open-source) ventures like Ardupilot (auto-pilot software) and cloud services, made available by some of the key players in the information technology sector (e.g. Microsoft, Amazon, Google, IBM, Oracle, etc.). The acquired data by such a system will allow the identification of pollution sources or the discharge and dispersion of contaminants along watercourses. State-of-the-art robotics and artificial intelligence technology are very powerful but remain difficult to implement by the less technically adept. Robotic monitoring systems are still a niche industry, with very high price tags/rates making them inaccessible to several interested parties who would undoubtedly benefit from said technologies This project aims to be a versatile, robust and reliable real-time water quality monitoring system, thus contributing to the management and protection of water resources, increasing security in the water supply of populations for a more efficient and sustainable management of resources. 


\section{Acknowledgement}

This work was supported by Centro2020, Portugal 2020 and European Union (EU) under the grants, CENTRO-01-0145-FEDER-024052E - Libélula: Mobile robotic surface water quality monitoring system.

\section{References}

1. Pule, M., Yahya, A., Chuma, J.: Wireless sensor networks: A survey on monitoring water quality. Journal of Applied Research and Technology, 15, 562 - 570 (2017)

2. Ballesteros-Gomez, A., Rubio, S.: Recent Advances in Environmental Analysis. Analytical Chemistry, Vol. 83, 12, 4579--4613 (2011)

3. Dunbabin, M., Marques, L.: Robots for Environmental monitoring: Significant advancements and applications. IEEE Robotics and Automation Magazine, 19, 24-39 (2012)

4. Xu, G., Shen, W., Wang, X.: Applications of Wireless Sensor Networks in Marine Environment Monitoring: A Survey. Sensors, 14, 16932-16954 (2014)

5. Ferreira, H., Almeida, C., Martins, A., Almeida, J., Dias, N., Dias, A.: Autonomous bathymetry for risk assessment with ROAZ robotic surface vehicle. In: Proceedings of the OCEANS 2009 IEEE conference, pp. 1--6. IEEE Press, New York (2009)

6. Raimundo, D., Leite, T., Silva, A.: Projeto de um Veículo Autónomo de Superfície para Monitorização de Águas Fluviais. EECS Project, IPT, Tomar (2016)

7. ArduPilot Open Source Autopilot, http://ardupilot.org

8. Bassiliades, N., Antoniades, I., Hatzikos, E., Vlahavas, I., Koutitas, G.: An Intelligent System for Monitoring and Predicting Water Quality. In: Proceedings of the European conference towards environment, pp. 534--542, Prague (2009)

9. Bhat, S., Meraj, G., Yaseen, S., K. Pandit, A.: Statistical Assessment of Water Quality Parameters for Pollution Source Identification in Sukhnag Stream: An Inflow Stream of Lake Wular (Ramsar Site). Journal of Ecosystems, 10.1155/2014/898054, Kashmir Himalaya (2014)

10. Mission Planner Documentation, http://ardupilot.org/planner/index.html

11. Thiyagarajan, K., Pappu, S., Vudatha, P., Niharika, A.V.: Intelligent IoT Based Water Quality Monitoring System. International Journal of Applied Engineering Research. 12. 5447 (2017) 